\title{
Assessment of metrics in next-generation sequencing experiments for use in core-genome multilocus sequence type
}

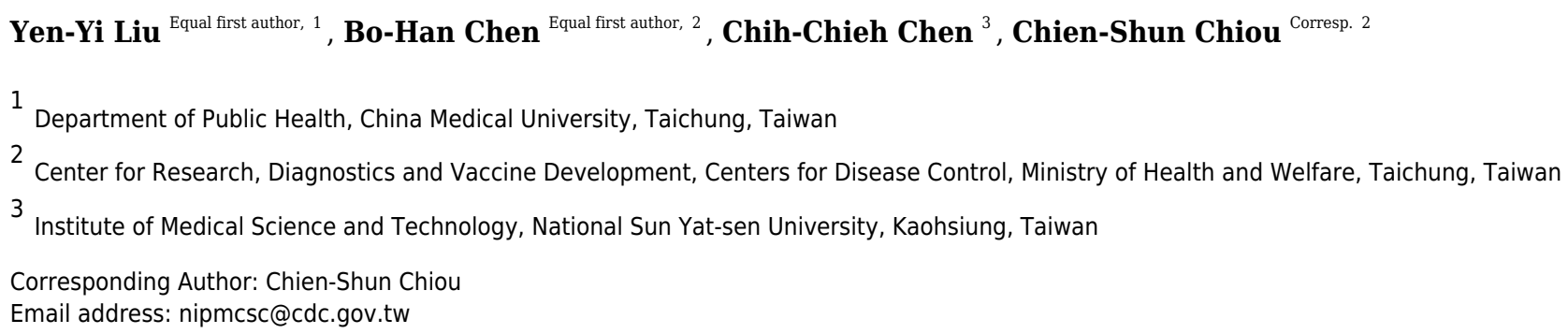

With the reduction in the cost of next-generation sequencing, whole-genome sequencing (WGS)-based methods such as core-genome multilocus sequence type (cgMLST) have been widely used. However, gene-based methods are required to assemble raw reads to contigs, thus possibly introducing errors into assemblies. Because the robustness of cgMLST depends on the quality of assemblies, the results of WGS should be assessed (from sequencing to assembly). In this study, we investigated the robustness of different read lengths, read depths, and assemblers in recovering genes from reference genomes. Different combinations of read lengths and read depths were simulated from the complete genomes of three common food-borne pathogens: Escherichia coli, Listeria monocytogenes, and Salmonella enterica. We found that the quality of assemblies was mainly affected by read depth, irrespective of the assembler used. In addition, we suggest several cutoff values for future cgMLST experiments. Furthermore, we recommend the combinations of read lengths, read depths, and assemblers that can result in a higher cost/performance ratio for cgMLST. 


\section{Assessment of metrics in next-generation sequencing experiments for use in}

\section{2 core-genome multilocus sequence type}

3 Yen-Yi Liu" ${ }^{1 \#}$, Bo-Han Chen", Chih-Chieh Chen ${ }^{3}$, Chien-Shun Chiou ${ }^{2 *}$

$4{ }^{1}$ Department of Public Health, China Medical University, Taichung, Taiwan.

$5 \quad{ }^{2}$ Center for Research, Diagnostics and Vaccine Development, Centers for Disease Control,

6 Ministry of Health and Welfare, Taichung, Taiwan,

7 Institute of Medical Science and Technology, National Sun Yat-sen University, Kaohsiung,

8 80424, Taiwan

9

10 Keywords: Molecular typing, Next generation sequencing (NGS), core-genome multilocus

11 sequence typing (cgMLST)

13 \# YY Liu and BH Chen contributed equally to this work.

14 *Address correspondence to: Chien-Shun Chiou, Central Region Laboratory, Center for

15 Research, Diagnostics and Vaccine Development, Centers for Disease Control, Ministry of

16 Health and Welfare; No. 20 Wen-Sin South 3rd Road, Taichung 40855, Taiwan. Phone: +8864

17 24750452. FAX: +886 4 24750474. E-mail: nipmcsc@cdc.gov.tw

19 Email addresses:

YYL: ethan.liu@.cmu.edu.tw 
CSC: nipmcsc@edc.gov.tw

\section{ABSTRACT}

26 With the reduction in the cost of next-generation sequencing, whole-genome sequencing

27 (WGS)-based methods such as core-genome multilocus sequence type (cgMLST) have been

28 widely used. However, gene-based methods are required to assemble raw reads to contigs, thus

29 possibly introducing errors into assemblies. Because the robustness of cgMLST depends on the

30 quality of assemblies, the results of WGS should be assessed (from sequencing to assembly). In

31 this study, we investigated the robustness of different read lengths, read depths, and assemblers

32 in recovering genes from reference genomes. Different combinations of read lengths and read

33 depths were simulated from the complete genomes of three common food-borne pathogens:

34 Escherichia coli, Listeria monocytogenes, and Salmonella enterica. We found that the quality of

35 assemblies was mainly affected by read depth, irrespective of the assembler used. In addition, we

36 suggest several cutoff values for future cgMLST experiments. Furthermore, we recommend the

37 combinations of read lengths, read depths, and assemblers that can result in a higher

38 cost/performance ratio for cgMLST. 
41 With the reduction in the cost of next-generation sequencing (NGS), whole-genome sequencing

42 (WGS)-based methods are being widely used in genomic epidemiology to characterize bacterial

43 pathogens and perform strain typing (Deng et al. 2016; Fratamico et al. 2016; Lindsey et al.

44 2016). Multilocus sequence type (MLST) genotyping (Maiden et al. 1998) has been used for

45 many years for cross-laboratory comparison and outbreak investigation among closely-related

46 strains. Core-genome MLST (cgMLST), an advanced version of MLST genotyping, is a

47 genome-wide gene-by-gene comparison approach (Maiden et al. 2013) that has been successfully

48 used for detecting disease clusters and investigating outbreaks (Barkley et al. 2016; de Been et

49 al. 2015; Jackson et al. 2016). Several websites and databases, such as PubMLST.org (Jolley et

50 al. 2018) and Pathogenwatch (https://pathogen.watch/), that are funded by large companies and

51 governments have been using cgMLST. Because of the increasing significance of cgMLST in the

52 field of epidemiology, evaluating its robustness is crucial. Bo Segerman (Segerman 2020) has

53 been reviewed sequencing technologies and assembly methods for the bacterial surveillance and

54 RefSeq Genome Database. He found that Illumina sequencers were the mostly used sequencing

55 platforms and SPAdes, SKESA and CLC were the most popular assemblers. Based on Bo

56 Segerman's findings, we designed a metric "number of core genes unrecalled" to find out the

57 minimum sequencing depth/coverage for SPAdes, SKESA and CLC at read lengths with 150bp

58 and $250 \mathrm{bp}$, which were common in Illumina platforms, to recover the most completely "core

59 gene alleles" (i.e. not only gene locus but also nucleotide sequence of such gene locus needed to

60 be the same). The idea of metric "core gene unrecalled" was from the benchmarking metrics of

61 genome assemblies (i.e. Contiguity, Correctness and Completeness) suggested by Jose Arturo

62 Molina Mora (Molina-Mora et al. 2020) with the scale from genome level down to gene level. 
63 Besides, because the genes order within a genome does not influence the generated cgMLST

64 profile, we only consider the correctness and completeness of core genes. Therefore, our

65 designed metric "number of core genes unrecalled" could fully reflect the quality of cgMLST

66 profiles. Since the sequencing read length, read depth, and assembler might substantially affect

67 cgMLST results, we investigated the effect of these factors on cgMLST results. In this study, we

68 simulated different read depths of different lengths from four common food-borne pathogens,

69 namely, Escherichia coli, Listeria monocytogenes, and Salmonella enterica, and performed

70 assembling by using different assemblers to determine the minimum read depths required under

71 different situations (i.e., different combinations of read lengths, read depths, and assemblers).

72 The minimum read depths determined in this study might help researchers in estimating the

73 depths before conducting cgMLST studies.

74 METHODS AND MATERIALS

75 To evaluate the minimum read depth required for recalling genes, we simulated read sets with

76 different read depths from complete reference genomes downloaded from NCBI. Three food-

77 borne pathogens were tested: E. coli, L. monocytogenes, and S. enterica. Different assemblers

78 and read lengths were included in the evaluation. The experiments were repeated three times to

79 ensure the robustness of the results.

80 Bacterial genomes used for evaluation

81 IAI39 (Touchon et al. 2009), EGD-e (Toledo-Arana et al. 2009), and LT2 (McClelland et al.

82 2001), which were the NCBI reference genomes with complete assembly level, were selected for

83 representing E. coli, L. monocytogenes, and S. enterica, respectively. The art_illumina simulator

84 of ART simulation toolkit (Huang et al. 2012) was used to generate pseudo reads with different 
85 read lengths and read depths from the selected four complete genomes. The command of

86 art_illumina used in this research is “art_illumina $-\mathrm{p}-$ na - ss MSv3 $-\mathrm{i}<$ reference $>-1<$ read

87 length $>-\mathrm{f}<$ depth $>-$ m $<$ read length $+50>-$ s $10-\mathrm{o}<$ path/file $>$.

\section{Metrics used for evaluation}

89 The metric "number of core genes unrecalled (i.e. number of void cgMLST loci or error called

90 cgMLST alleles)" was designed for finding out the minimum sequencing depth/coverage for

91 SPAdes, SKESA and CLC at common Illumina produced read lengths of 150bp and 250bp to

92 recover the most completely "core gene alleles", which means exactly the same with core gene

93 sequences. In addition, because the genes order within a genome does not influence the

94 generated cgMLST (Maiden et al. 2013) profile, we only consider the correctness and

95 completeness of core genes. Therefore, the quality of cgMLST profiles could be reflected

96 through evaluating "number of core genes unrecalled". The cgMLST allele calling was achieved

97 by using BENGA server (Chen et al. 2021).

98 Evaluation of the minimum sequencing depths achieving stable number of core genes

99 unrecalled by using different assemblers for different read lengths

100 To evaluate read depths required for different read lengths, we simulated 14 sequencing depths

101 or coverages $(10 \times, 20 \times, 30 \times, 40 \times, 50 \times, 60 \times, 70 \times, 80 \times, 90 \times, 100 \times, 200 \times, 300 \times, 400 \times$, and $500 \times)$

102 from $S$. enterica LT2, E. coli IAI39, and L. monocytogenes EGD-e. Each simulated read set was

103 assembled using SPAdes (Bankevich et al. 2012), CLC Genomics Workbench v10.1.1 (CLC),

104 and SKESA (Souvorov et al. 2018), and the resulting contigs were compared with the original

105 complete genomes. The reads assembly settings for the three assemblers were listed in Table S1.

106 All genes were predicted using the Prodigal program (Hyatt et al. 2010). The "gene recalled" 
107 was defined as the predicted gene in the assembly showed a $100 \%$ match with the predicted gene

108 in the original complete genome. The three assemblers used for the read lengths of 150 and 250

$109 \mathrm{bp}$ were compared to determine the minimum coverage needed to recover the maximum genes

110 for different read lengths, regardless of assemblers. Deviations in the number of unrecalled genes

111 for the same assembler, read depth, and read length can be caused due to the stochastic procedure

112 of read simulation.

113 Evaluation of minimum sequencing depths for the three common food-borne pathogens $(S$.

114 enterica, $E$. coli, L. monocytogenes) based on real sequenced data

115 To reflect the real sequenced reads condition, we picked up genomes both having raw reads data

116 in SRA database and assembled genomes with complete level in GenBank for further evaluation.

117 The complete assembled genome from GenBank can be used as the reference for evaluating the

118 raw reads assembling from SRA. We sampled different read depths (i.e. $10 \times, 20 \times, 30 \times, 40 \times$,

$11950 \times, 60 \times, 70 \times, 80 \times, 90 \times$, and $100 \times)$ using Seqtk

120 (https://github.com/lh3/seqtk/blob/master/README.md) from the real sequenced reads data of

121 S. enterica (SRR5866640 for $150 \mathrm{bp}$ and SRR6929558 for $250 \mathrm{bp),} \mathrm{E.} \mathrm{coli} \mathrm{(SRR6924239} \mathrm{for} 150$

122 bp and SRR3205757 for 250 bp), and L. monocytogenes (SRR3089759 for $150 \mathrm{bp}$ and

123 SRR6347431 for $250 \mathrm{bp}$ ). To investigate the minimum read depth required for achieving the

124 stable core genes unrecalled of real sequenced reads data, we picked up the relevant (i.e. having

125 the same BioSample Accession Number) assembled genomes with complete level as the

126 reference for the evaluation. The relevant assembled genomes are $S$. enterica (CP023508.1 for

$127150 \mathrm{bp}$ and CP036165.1 for $250 \mathrm{bp}$ ), E. coli (CP029239.1 for $150 \mathrm{bp}$ and CP034799.1 for 250

128 bp), and L. monocytogenes (CP013919.1 for $150 \mathrm{bp}$ and CP025565.1 for $250 \mathrm{bp}$ ). The command

129 for performed Seqtk is "seqtk sample -s [seed] [input] [fraction] > [output]". 
130 Estimation of the sequencing depth for three commonly used assemblers for completing the

131 assembly process in a linear time

132 To evaluate the running time of SPAdes, CLC, and SKESA assemblers, we determined the time

133 required for assembling simulated reads with a read depth of $10 \times, 20 \times, 30 \times, 40 \times, 50 \times, 60 \times, 70 \times$,

$13480 \times, 90 \times$, and $100 \times$. The read length of $250 \mathrm{bp}$ was chosen for testing. The server equipped with

135 Intel Xeon CPU E7-4830 v4 2.00 GHz was used for the evaluation. The experiment was

136 performed under the condition of eight threads in a 32-GB RAM computation environment. Wall

137 time was used to evaluate the running time.

138 RESULTS

139 We evaluated 14 sequencing depths or coverages $(10 \times, 20 \times, 30 \times, 40 \times, 50 \times, 60 \times, 70 \times, 80 \times, 90 \times$,

$140100 \times, 200 \times, 300 \times, 400 \times$, and $500 \times$ ) for determining the assembly quality. The number of

141 unrecalled genes from the reference genomes of $S$. enterica LT2, E. coli IAI39, and $L$.

142 monocytogenes EGD-e represented the assembly quality. Three commonly used assemblers,

143 namely SPAdes, CLC, and SKESA, were applied to run the tests. Two read lengths, $150 \mathrm{bp}$ and

$144250 \mathrm{bp}$, representing the widely used sequencing read lengths in Illumina HiSeq and Illumina

145 MiSeq platforms, respectively, were evaluated.

146 Minimum sequencing depth achieving stable number of core genes unrecalled for different

147 assemblers by using different read lengths

148 As shown in Figure 1, a sequencing coverage of $60 \times$ might be a safe choice irrespective of the

149 assembler and read length. We observed that the SPAdes assembler required $30 \times$ read depths

150 (irrespective of whether the read length of 150 or $250 \mathrm{bp}$ was used) to achieve minimum depth of 
151 the stable core genes unrecalled compared with CLC and SKESA that required read depths of

$15240 \times \sim 60 \times$. Because the read lengths of 150 and 250 bp are mainly used in Illumina platforms,

153 we evaluated the minimum sequencing coverage required for these two read lengths. As shown

154 in Figure 1, sequencing coverages of at least $60 \times$ and $50 \times$ were required for the read lengths of

155150 and $250 \mathrm{bp}$, respectively, for assembly to achieve the stable core genes unrecalled

156 irrespective of the assemblers used (i.e., SPAdes, CLC, and SKESA). Regarding assemblers, we

157 observed that SPAdes was not considerably affected by the read length and required a read depth

158 of only $30 \times$ to recover reference genes. However, CLC and SKESA required a read depth of at

159 least $40 \times-50 \times$ and $50 \times-60 \times$, respectively, to achieve assembly quality similar to that obtained

160 using SPAdes.

161 Plausible sequencing depths for the three commonly used assemblers to complete the

162 procedure in linear time

163 As shown in Figure S1, the assembly time required by SKESA and CLC did not change even at a

164 sequence depth of $500 \times$. However, for SPAdes, the assembly time increased according to the

165 sequence depth, particularly when it was more than $100 \times$. In addition, the assembly time was not

166 affected by the read length for SKESA and CLC; however, for SPAdes, a read length of $150 \mathrm{bp}$

167 required more time for assembly than a read length of $250 \mathrm{bp}$.

\section{Suggested minimum sequencing depths for achieving stable number of core genes}

169 unrecalled of four common food-borne pathogens (S. enterica, E. coli, L. monocytogenes)

170 based on simulation reads

171 All the aforementioned evaluation results were obtained from simulated S. enterica LT2, E. coli,

172 and L. monocytogenes reads (shown in Table 1). The minimum required depth tended to be 
173 similar among the four tested species, with a minimum depth of $30 \times$ for SPAdes and $40 \times-50 \times$

174 for CLC at a read length of $150 \mathrm{bp}$ and $30 \times$ for SPAdes and $40 \times-50 \times$ for CLC at a read length of

175250 bp. Compared with SPAdes and CLC, the minimum read coverage for SKESA was $40 \times-60 \times$

176 at a read length of $150 \mathrm{bp}$ and $40 \times-50 \times$ at a read length of $250 \mathrm{bp}$. The minimum coverage

177 (depth) of SPAdes, CLC, and SKESA at different read lengths and sequence coverages are

178 highlighted in gray (shown in Table 1).

179 Suggested minimum sequencing depths for the three common food-borne pathogens $(S$.

180 enterica, E. coli, L. monocytogenes) based on real sequenced data

181 The results of the minimum read depth sampled from real sequenced reads required for achieving

182 the stable number of core genes unrecalled were shown in Table 2. The results were similar to

183 those obtained for simulation data with a minimum depth of $30 \times$ for SPAdes and $30 \times-40 \times$ for

184 CLC assemblers at a read length of 150 bp and $20 \times-40 \times$ for SPAdes and $20 \times-50 \times$ for CLC at a

185 read length of $250 \mathrm{bp}$. Compared with SPAdes and CLC, the minimum read coverage for

186 SKESA was $50 \times-70 \times$ at a read length of $150 \mathrm{bp}$ and $50 \times-70 \times$ for a read length of $250 \mathrm{bp}$. The

187 reads QC were performed by using FastQC

188 (https://www.bioinformatics.babraham.ac.uk/projects/fastqc/), and the "Per sequence quality scores"

189 and "Sequence Length Distribution" from QC reports were shown in Figure S2.

\section{DISCUSSION}

191 In our evaluation, we applied an index "genes called" to represent the assembly quality. Because

192 read sets were simulated from a complete genome, the number of "genes called" can directly

193 represent the quality of assemblies. Because the number of genes called can indicate the

194 completeness of a "pan genome," which covers "core genes," we used genes called as our 
195 evaluation index. The three most important factors in NGS were evaluated: read depth, read

196 length, and assembler. We found that an assembler was the most crucial factor that affected the

197 quality of assemblies, especially at a low read depth. For low read depths $(20 \times-30 \times)$, SPAdes

198 outperformed CLC and SKESA with an error rate of $<2.0 \%$, although the performance of CLC

199 was close to that of SPAdes. Compared with SPAdes and CLC, SKESA usually required 40×-

$20050 \times$ to reach an error rate of $<2.0 \%$. Although SPAdes demonstrated the highest performance, its

201 running time considerably increased with the read depth, especially when the depth was $>100 \times$.

202 No difference in results was observed between long reads (250 bp) and short reads (150 bp) for

203 SPAdes and CLC; however, SKESA required a larger depth to assemble short reads to reach an

204 error rate of $<2.0 \%$. In addition, to investigate if some similarity sharing among unrecalled genes

205 at even high sequencing depth, we analyzed the unrecalled core genes of S. enterica, E. coli and

206 L. monocytogenes at depth $100 \times$, and no commonality among these genes was found. The

207 unrecalled core genes at depth $100 \times$ from Table 1 are listed in Table S2 (the union of the triple

208 repeat are listed). In summary, we recommend sequencing at a read depth of $30 \times-50 \times$ and a read

209 length of $250 \mathrm{bp}$ by using SPAdes as the assembler to maintain a balance of cost/pay ratio in

210 cgMLST.

\section{AUTHOR CONTRIBUTIONS}

213 Conceived and designed the experiments: YYL, BHC and CSC. Performed the experiments and

214 analyzed the data: YYL and BHC. Wrote the paper: YYL, CCC, CSC. 


\section{COMPETING INTERESTS}

216 The authors declare that they have no competing interests.

\section{FUNDING}

218 This study was supported by the Ministry of Health and Welfare, Taiwan with Grant No.

219 MOHW106-CDC-C-315-114712.

220

221

222

223

224

225

226

227

228

229

230

231

232

233

234

235

236

237

238

239

240

241

242

243

244

\section{REFERENCES}

Bankevich A, Nurk S, Antipov D, Gurevich AA, Dvorkin M, Kulikov AS, Lesin VM, Nikolenko SI, Pham S, Prjibelski AD, Pyshkin AV, Sirotkin AV, Vyahhi N, Tesler G, Alekseyev MA, and Pevzner PA. 2012. SPAdes: a new genome assembly algorithm and its applications to single-cell sequencing. J Comput Biol 19:455-477. $10.1089 / \mathrm{cmb} .2012 .0021$

Barkley JS, Gosciminski M, and Miller A. 2016. Whole-Genome Sequencing Detection of Ongoing Listeria Contamination at a Restaurant, Rhode Island, USA, 2014. Emerg Infect Dis 22:1474-1476. 10.3201/eid2208.151917

Chen YS, Tu YH, Chen BH, Liu YY, Hong YP, Teng RH, Wang YW, and Chiou CS. 2021. cgMLST@Taiwan: A web service platform for Vibrio cholerae cgMLST profiling and global strain tracking. J Microbiol Immunol Infect. 10.1016/j.jmii.2020.12.007

de Been M, Pinholt M, Top J, Bletz S, Mellmann A, van Schaik W, Brouwer E, Rogers M, Kraat Y, Bonten M, Corander J, Westh H, Harmsen D, and Willems RJ. 2015. Core Genome Multilocus Sequence Typing Scheme for High- Resolution Typing of Enterococcus faecium. J Clin Microbiol 53:3788-3797. 10.1128/JCM.01946-15

Deng X, den Bakker HC, and Hendriksen RS. 2016. Genomic Epidemiology: Whole-GenomeSequencing-Powered Surveillance and Outbreak Investigation of Foodborne Bacterial Pathogens. Annu Rev Food Sci Technol 7:353-374. 10.1146/annurev-food-041715033259

Fratamico PM, DebRoy C, Liu Y, Needleman DS, Baranzoni GM, and Feng P. 2016. Advances in Molecular Serotyping and Subtyping of Escherichia coli. Front Microbiol 7:644. 10.3389/fmicb.2016.00644

Huang W, Li L, Myers JR, and Marth GT. 2012. ART: a next-generation sequencing read simulator. Bioinformatics 28:593-594. 10.1093/bioinformatics/btr708 
245

274

275

276

277

278

279

280

281

282

Hyatt D, Chen GL, Locascio PF, Land ML, Larimer FW, and Hauser LJ. 2010. Prodigal: prokaryotic gene recognition and translation initiation site identification. $B M C$ Bioinformatics 11:119. 10.1186/1471-2105-11-119

Jackson BR, Tarr C, Strain E, Jackson KA, Conrad A, Carleton H, Katz LS, Stroika S, Gould LH, Mody RK, Silk BJ, Beal J, Chen Y, Timme R, Doyle M, Fields A, Wise M, Tillman G, Defibaugh-Chavez S, Kucerova Z, Sabol A, Roache K, Trees E, Simmons M, Wasilenko J, Kubota K, Pouseele H, Klimke W, Besser J, Brown E, Allard M, and Gerner-Smidt P. 2016. Implementation of Nationwide Real-time Whole-genome Sequencing to Enhance Listeriosis Outbreak Detection and Investigation. Clin Infect Dis 63:380-386. 10.1093/cid/ciw242

Jolley KA, Bray JE, and Maiden MCJ. 2018. Open-access bacterial population genomics: BIGSdb software, the PubMLST.org website and their applications. Wellcome Open Res 3:124. 10.12688/wellcomeopenres.14826.1

Lindsey RL, Pouseele H, Chen JC, Strockbine NA, and Carleton HA. 2016. Implementation of Whole Genome Sequencing (WGS) for Identification and Characterization of Shiga Toxin-Producing Escherichia coli (STEC) in the United States. Front Microbiol 7:766. 10.3389/fmicb.2016.00766

Maiden MC, Bygraves JA, Feil E, Morelli G, Russell JE, Urwin R, Zhang Q, Zhou J, Zurth K, Caugant DA, Feavers IM, Achtman M, and Spratt BG. 1998. Multilocus sequence typing: a portable approach to the identification of clones within populations of pathogenic microorganisms. Proc Natl Acad Sci U S A 95:3140-3145. 10.1073/pnas.95.6.3140

Maiden MC, Jansen van Rensburg MJ, Bray JE, Earle SG, Ford SA, Jolley KA, and McCarthy ND. 2013. MLST revisited: the gene-by-gene approach to bacterial genomics. Nat Rev Microbiol 11:728-736. 10.1038/nrmicro3093

McClelland M, Sanderson KE, Spieth J, Clifton SW, Latreille P, Courtney L, Porwollik S, Ali J, Dante M, Du F, Hou S, Layman D, Leonard S, Nguyen C, Scott K, Holmes A, Grewal N, Mulvaney E, Ryan E, Sun H, Florea L, Miller W, Stoneking T, Nhan M, Waterston R, and Wilson RK. 2001. Complete genome sequence of Salmonella enterica serovar Typhimurium LT2. Nature 413:852-856. 10.1038/35101614

Molina-Mora JA, Campos-Sanchez R, Rodriguez C, Shi L, and Garcia F. 2020. High quality 3C de novo assembly and annotation of a multidrug resistant ST-111 Pseudomonas aeruginosa genome: Benchmark of hybrid and non-hybrid assemblers. Sci Rep 10:1392. 10.1038/s41598-020-58319-6

Segerman B. 2020. The Most Frequently Used Sequencing Technologies and Assembly Methods in Different Time Segments of the Bacterial Surveillance and RefSeq Genome Databases. Front Cell Infect Microbiol 10:527102. 10.3389/fcimb.2020.527102

Souvorov A, Agarwala R, and Lipman DJ. 2018. SKESA: strategic k-mer extension for scrupulous assemblies. Genome Biol 19:153. 10.1186/s13059-018-1540-z

Peer] reviewing PDF | (2021:04:59837:2:0:NEW 20 Jun 2021) 
283 284 285 286 287

296

Toledo-Arana A, Dussurget O, Nikitas G, Sesto N, Guet-Revillet H, Balestrino D, Loh E, Gripenland J, Tiensuu T, Vaitkevicius K, Barthelemy M, Vergassola M, Nahori MA, Soubigou G, Regnault B, Coppee JY, Lecuit M, Johansson J, and Cossart P. 2009. The Listeria transcriptional landscape from saprophytism to virulence. Nature 459:950-956. 10.1038 /nature 08080

Touchon M, Hoede C, Tenaillon O, Barbe V, Baeriswyl S, Bidet P, Bingen E, Bonacorsi S, Bouchier C, Bouvet O, Calteau A, Chiapello H, Clermont O, Cruveiller S, Danchin A, Diard M, Dossat C, Karoui ME, Frapy E, Garry L, Ghigo JM, Gilles AM, Johnson J, Le Bouguenec C, Lescat M, Mangenot S, Martinez-Jehanne V, Matic I, Nassif X, Oztas S, Petit MA, Pichon C, Rouy Z, Ruf CS, Schneider D, Tourret J, Vacherie B, Vallenet D, Medigue C, Rocha EP, and Denamur E. 2009. Organised genome dynamics in the Escherichia coli species results in highly diverse adaptive paths. PLoS Genet 5:e1000344. 10.1371/journal.pgen.1000344 
FIGURE CAPTIONS

299 Figure 1. Estimation of the minimum read coverage required to achieve the stable core

300 genes unrecalled for assembling at a read length of 150 and 250 bp. Comparison of different

301 assemblers for the number of unrecalled genes from the reference genome (S. enterica LT2, E.

302 coli IAI39, and L. monocytogenes EGD-e) according to different simulated read coverages (10×,

$30320 \times, 30 \times, 40 \times, 50 \times, 60 \times, 70 \times, 80 \times, 90 \times, 100 \times, 200 \times, 300 \times, 400 \times$, and $500 \times)$ at read lengths of

304150 (left panel, A) and $250 \mathrm{bp}$ (right panel, B).

305 Figure S1. The estimation of the minimum read coverage for the running time of SPAdes,

306 CLC, and SKESA assemblers. Comparison of different read lengths for the assembling running

307 time according to different simulated read coverages among SPAdes, CLC, and SKESA for $S$.

308 enterica LT2.

309 Figure S2. The real sequenced reads QC performed by using FastQC. The "Per sequence

310 quality scores" and "Sequence Length Distribution" from QC reports of (A) S. enterica

311 (SRR5866640 for $150 \mathrm{bp}$ and SRR6929558 for $250 \mathrm{bp}$ ), (B) E. coli (SRR6924239 for $150 \mathrm{bp}$ and

312 SRR3205757 for 250 bp), and (C) L. monocytogenes (SRR3089759 for $150 \mathrm{bp}$ and SRR6347431

313 for $250 \mathrm{bp}$ ) were shown. 


\section{Figure 1}

Estimation of the minimum read coverage required to achieve the stable core genes unrecalled for assembling at a read length of 150 and $250 \mathrm{bp}$.

Comparison of different assemblers for the number of unrecalled genes from the reference genome (S. enterica LT2, E. coli IAI39, and L. monocytogenes EGD-e) according to different simulated read coverages $(10 x, 20 x, 30 x, 40 x, 50 x, 60 x, 70 x, 80 x, 90 x, 100 x, 200 x$, $300 \times, 400 \times$, and 500x) at read lengths of 150 (left panel, A) and $250 \mathrm{bp}$ (right panel, B). 
(A)

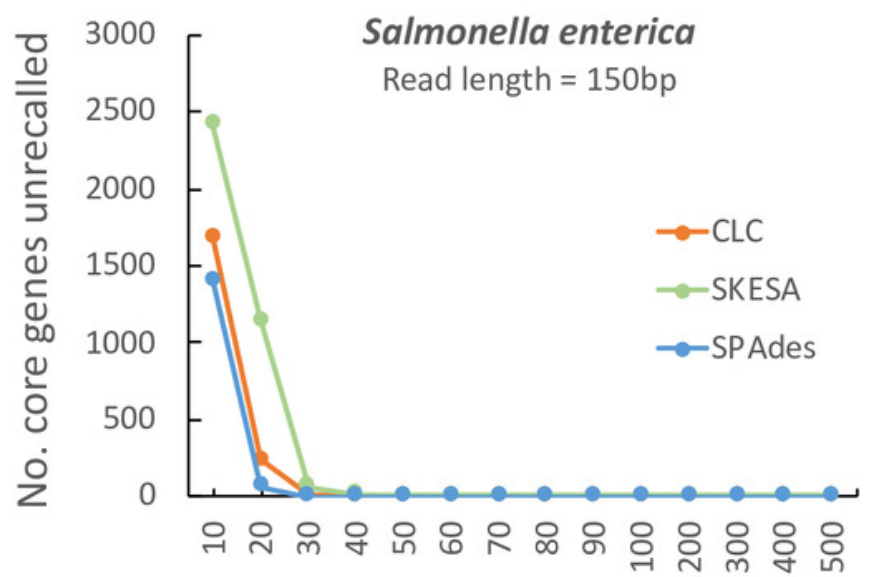

Depth

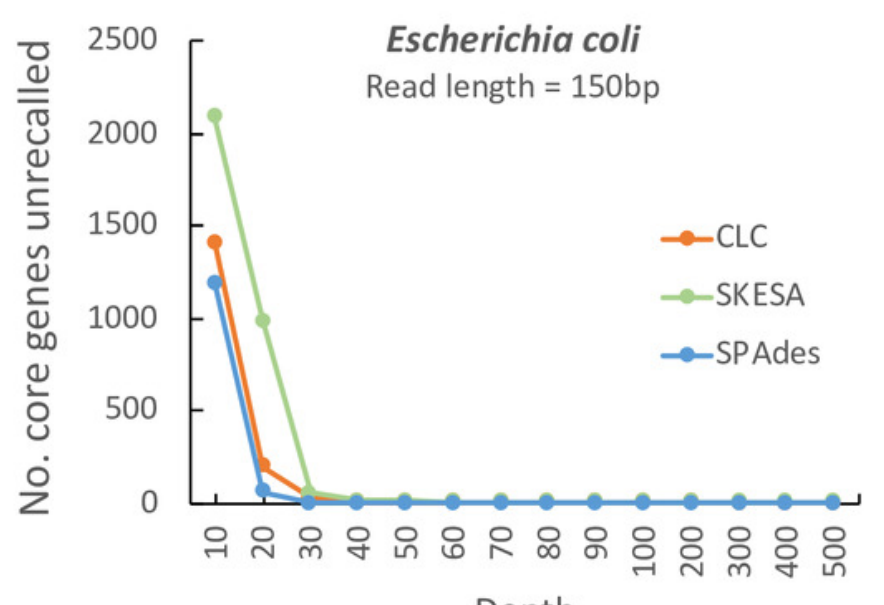

Depth

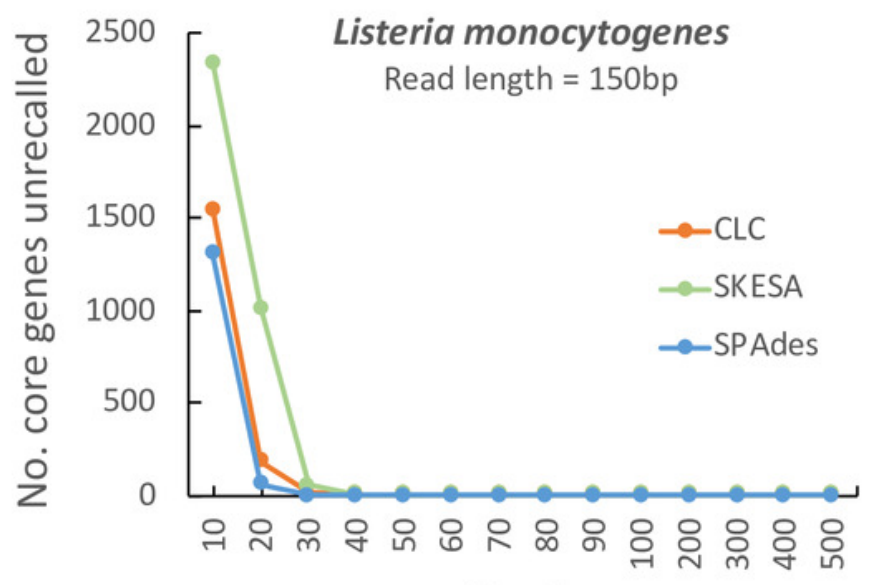

Depth
(B)

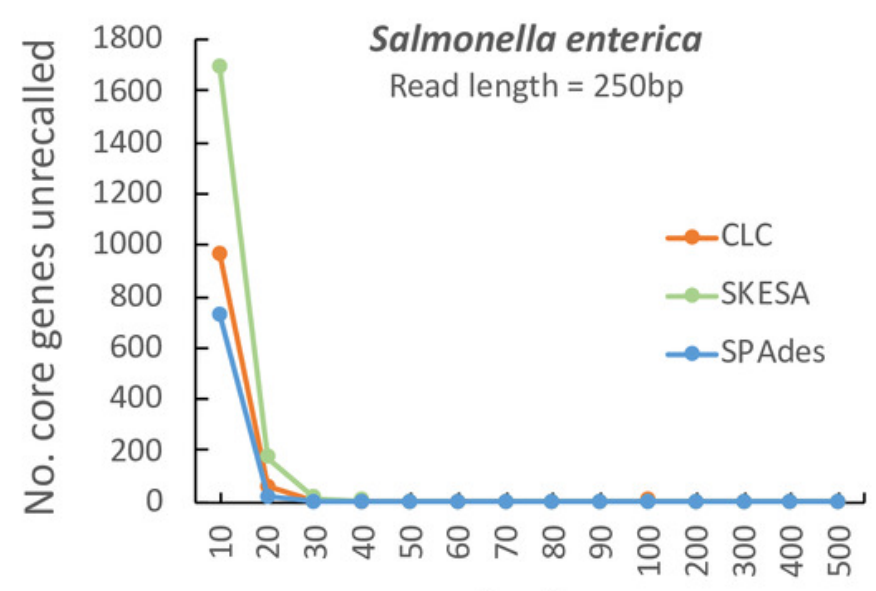

Depth

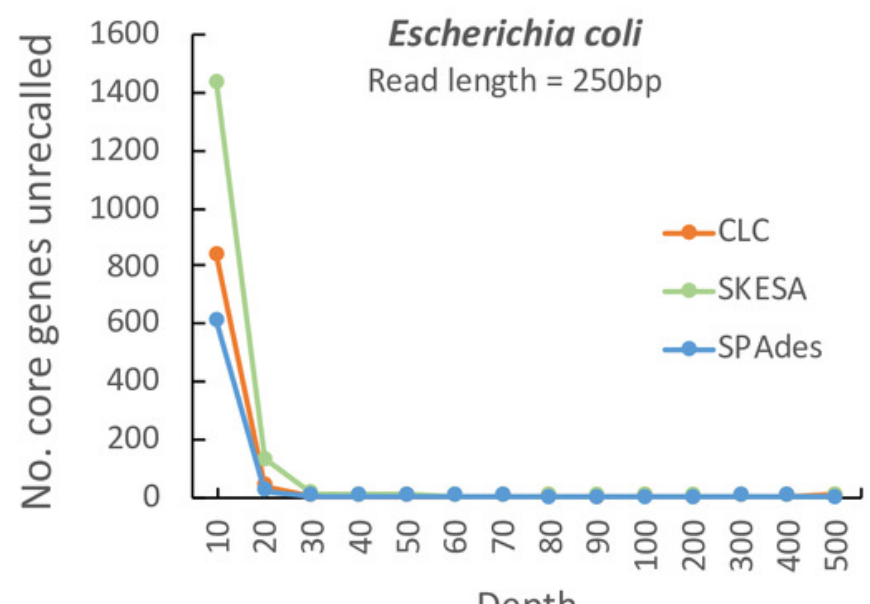

Depth

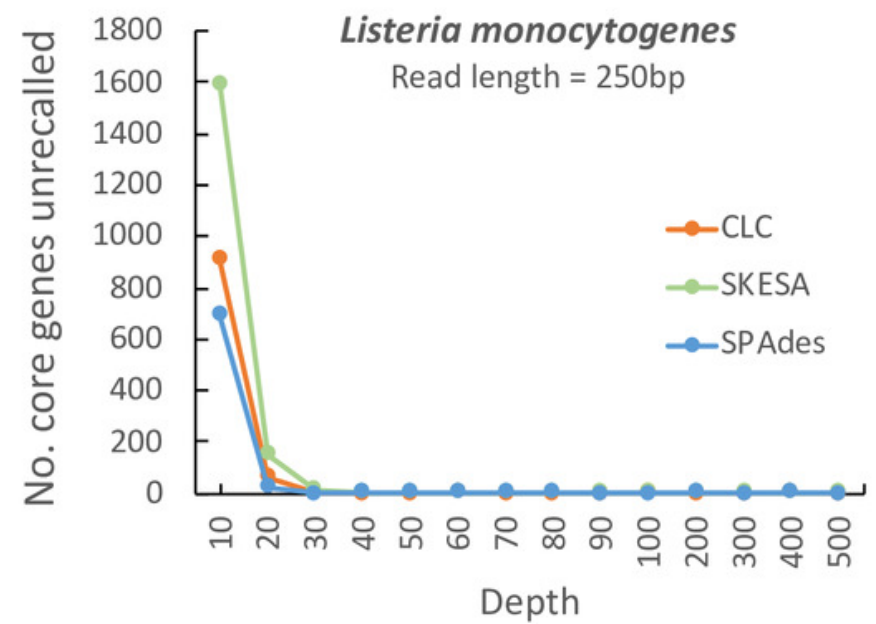




\section{Table $\mathbf{1}$ (on next page)}

The number of core genes unrecalled at each depth comparing for different assemblers based on simulated data.

The number of core genes unrecalled at each depth comparing for different assemblers based on simulated data. 
Table 1. The number of core genes unrecalled at each depth comparing for different assemblers based on simulated data.

\begin{tabular}{|c|c|c|c|c|c|c|c|c|c|c|c|c|}
\hline & \multirow[t]{2}{*}{ Read length } & \multirow[t]{2}{*}{ Assemblers } & \multicolumn{10}{|c|}{ Read depth } \\
\hline & & & $10 \times$ & $20 \times$ & $30 \times$ & $40 \times$ & $50 \times$ & $60 \times$ & $70 \times$ & $80 \times$ & $90 \times$ & $100 \times$ \\
\hline S. enterica (LT2) & $150 \mathrm{bp}$ & SPAdes & $1404-1419$ & $64-74$ & $3-4^{a}$ & $3-4$ & 3 & $3-4$ & $3-4$ & 3 & $3-4$ & $2-3$ \\
\hline \multirow[t]{5}{*}{$(\mathrm{Size}=4.9 \mathrm{Mb})$} & & CLC & $1680-1688$ & $241-243$ & $10-30$ & $3-12$ & 2 & 3 & $2-3$ & $1-3$ & $2-3$ & $2-4$ \\
\hline & & SKESA & $2382-2443$ & $1125-1157$ & $62-68$ & $13-14$ & $8-9$ & 5 & 5 & 5 & 5 & 5 \\
\hline & $250 \mathrm{bp}$ & SPAdes & $728-752$ & $15-27$ & $3-4$ & 2 & $1-2$ & $1-2$ & $1-2$ & $2-3$ & $1-2$ & $2-3$ \\
\hline & & CLC & $964-1025$ & $55-113$ & 5 & $1-2$ & $2-3$ & $2-3$ & 2 & 2 & $2-4$ & $2-5$ \\
\hline & & SKESA & $1692-1697$ & $169-175$ & $12-17$ & 3 & 2 & 2 & 2 & 2 & 2 & 2 \\
\hline L. monocytogens & $150 \mathrm{bp}$ & SPAdes & 1036 & 48 & 0 & 0 & 0 & 0 & 0 & 0 & 0 & 0 \\
\hline (IAI39) & & CLC & $1223-1226$ & $139-143$ & $3-5$ & 0 & 0 & 0 & 0 & 0 & 0 & 0 \\
\hline \multirow[t]{4}{*}{$(\mathrm{Size}=2.9 \mathrm{Mb})$} & & SKESA & 1839 & $790-791$ & 32 & 1 & $0-1$ & 1 & 1 & 1 & 1 & 1 \\
\hline & $250 \mathrm{bp}$ & SPAdes & $531-560$ & $12-21$ & $0-1$ & 0 & $0-1$ & 0 & $0-1$ & 0 & 0 & 0 \\
\hline & & CLC & $686-735$ & $36-66$ & $0-2$ & 0 & $0-2$ & 0 & 0 & 0 & 0 & 0 \\
\hline & & SKESA & $1217-1267$ & $111-126$ & $3-11$ & $1-3$ & 1 & 1 & 1 & 1 & 1 & $0-1$ \\
\hline E. coli (EGD-e) & $150 \mathrm{bp}$ & SPAdes & 1188 & 61 & 4 & 4 & $3-4$ & 4 & $3-5$ & 3 & $2-3$ & $3-4$ \\
\hline \multirow[t]{5}{*}{$(\mathrm{Size}=4.6 \mathrm{Mb})$} & & CLC & $1411-1416$ & $197-202$ & $30-32$ & $5-8$ & $5-6$ & $4-5$ & 5 & $2-6$ & 5 & $5-6$ \\
\hline & & SKESA & 2089 & 989 & 54 & 14 & $9-11$ & 8 & 8 & $7-8$ & 7 & 7 \\
\hline & $250 \mathrm{bp}$ & SPAdes & $611-629$ & $21-22$ & 4 & 3 & $2-3$ & 3 & 3 & $2-3$ & 2 & 2 \\
\hline & & CLC & $811-839$ & $40-60$ & $4-6$ & $3-4$ & $3-4$ & $2-4$ & $3-4$ & $4-5$ & 2 & 4 \\
\hline & & SKESA & $1403-1432$ & $132-135$ & $13-14$ & $7-8$ & 7 & $6-7$ & $6-7$ & 6 & 6 & 6 \\
\hline
\end{tabular}

\footnotetext{
${ }^{a}$ The gray fill represents the minimum read depth needed to achieve the stable number of core genes unrecalled for the combing of different read lengths and
} assemblers. 


\section{Table 2 (on next page)}

The number of core-gene differences between assembly and the reference genome in each depth comparing for different assemblers based on real sequenced data.

The number of core-gene differences between assembly and the reference genome in each depth comparing for different assemblers based on real sequenced data. 
Table 2. The number of core-gene differences between assembly and the reference genome in each depth comparing for

2 different assemblers based on real sequenced data.

\begin{tabular}{|c|c|c|c|c|c|c|c|c|c|c|c|c|}
\hline & \multirow[t]{2}{*}{ Read length } & \multirow[t]{2}{*}{ Assemblers } & \multicolumn{10}{|c|}{ Read depth } \\
\hline & & & $10 \times$ & $20 \times$ & $30 \times$ & $40 \times$ & $50 \times$ & $60 \times$ & $70 \times$ & $80 \times$ & $90 \times$ & $100 \times$ \\
\hline \multirow[t]{6}{*}{ S. enterica } & $150 \mathrm{bp}$ & SPAdes & $180-206$ & $8-11$ & $7-8^{a}$ & $7-8$ & $7-8$ & $7-8$ & $7-8$ & $7-7$ & $6-8$ & $7-8$ \\
\hline & (CP023508.1) & CLC & $384-439$ & $13-17$ & $7-8$ & $7-8$ & $7-9$ & $7-9$ & $7-7$ & $7-9$ & $7-9$ & $7-7$ \\
\hline & SRR5866640 & SKESA & $2669-2864$ & $871-1177$ & $89-652$ & $24-55$ & $13-14$ & $12-13$ & $13-14$ & 13 & $13-14$ & 13 \\
\hline & 250 bp & SPAdes & $185-214$ & $11-15$ & $8-9$ & $8-10$ & $8-9$ & $8-9$ & 8 & $8-9$ & $8-9$ & 8 \\
\hline & (CP036165.1) & CLC & $338-392$ & $12-28$ & 10 & $7-11$ & $7-8$ & $5-9$ & $8-9$ & $5-8$ & $8-9$ & $7-8$ \\
\hline & SRR6929558 & SKESA & $2373-2570$ & $874-885$ & $120-145$ & $16-22$ & $11-12$ & $10-11$ & 10 & 10 & $10-11$ & 10 \\
\hline \multirow[t]{6}{*}{ L. monocytogens } & 150 bp & SPAdes & $376-423$ & $12-14$ & $0-1$ & 0 & 0 & 0 & 0 & 0 & 0 & 0 \\
\hline & (CP013919.1) & CLC & $593-658$ & $41-60$ & $3-5$ & $0-2$ & $0-1$ & 1 & $0-1$ & 1 & 1 & $0-1$ \\
\hline & SRR3089759 & SKESA & 2000-2059 & $1144-1192$ & $660-671$ & $55-130$ & $6-14$ & $2-3$ & $1-3$ & $1-2$ & 1 & 1 \\
\hline & 250 bp & SPAdes & $176-200$ & $7-14$ & $1-2$ & $0-2$ & 0 & 0 & $0-1$ & 0 & 0 & 0 \\
\hline & (CP025565.1) & CLC & $325-349$ & $40-55$ & $7-12$ & $3-8$ & $0-1$ & $0-3$ & 1 & $0-1$ & 0 & 0 \\
\hline & SRR6347431 & SKESA & $1521-1620$ & $597-612$ & $101-125$ & $22-39$ & $6-7$ & $3-5$ & $1-3$ & $0-2$ & $0-3$ & $1-2$ \\
\hline \multirow[t]{6}{*}{ E. coli } & $150 \mathrm{bp}$ & SPAdes & $97-122$ & $11-12$ & $9-12$ & $9-10$ & 10 & $9-11$ & $9-10$ & $9-10$ & 9 & $9-10$ \\
\hline & (CP029239.1) & CLC & $217-234$ & $21-26$ & $12-17$ & $11-14$ & $11-13$ & $11-13$ & 11 & $11-12$ & 11 & $11-13$ \\
\hline & SRR6924239 & SKESA & $2017-2137$ & $905-922$ & $468-518$ & $34-60$ & $17-21$ & $12-14$ & 13 & $11-12$ & 12 & 12 \\
\hline & $250 \mathrm{bp}$ & SPAdes & $37-56$ & 4 & 4 & 4 & 4 & 4 & 4 & 4 & 4 & 4 \\
\hline & (CP034799.1) & CLC & $76-99$ & 6 & $5-6$ & $5-6$ & $5-6$ & $4-6$ & $5-6$ & $5-6$ & $5-7$ & $6-7$ \\
\hline & SRR3205757 & SKESA & $1651-1780$ & $449-553$ & $22-30$ & $7-8$ & 6 & 6 & 6 & 6 & 6 & 6 \\
\hline
\end{tabular}

3 a The gray fill represents the minimum read depth needed to achieve the stable number of core genes unrecalled for the combing of different read lengths and assemblers. 
